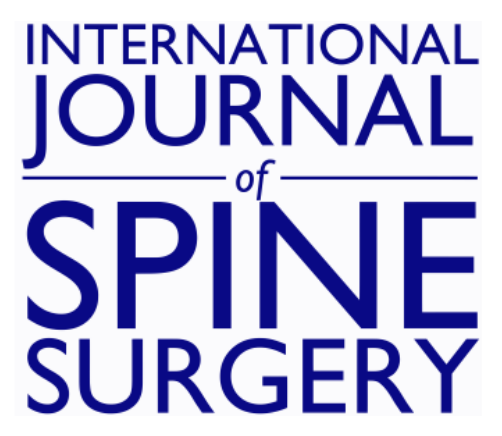

\title{
In Vitro Biomechanics of Human Cadaveric Cervical Spines With Mature Fusion
}

Anna G. U. Sawa, Bernardo de Andrada Pereira, Nestor G. Rodriguez-Martinez, Phillip M. Reyes, Brian P. Kelly and Neil R. Crawford

Int J Spine Surg published online 22 September 2021

http://ijssurgery.com/content/early/2021/09/20/8114

This information is current as of April 26, 2023.

Email Alerts Receive free email-alerts when new articles cite this article. Sign up at:

http://ijssurgery.com/alerts 


\title{
In Vitro Biomechanics of Human Cadaveric Cervical Spines With Mature Fusion
}

\author{
ANNA G. U. SAWA, MS, BERNARDO DE ANDRADA PEREIRA, MD, NESTOR G. RODRIGUEZ- \\ MARTINEZ, MD, PHILLIP M. REYES, BSE, BRIAN P. KELLY, PHD, NEIL R. CRAWFORD, PHD \\ Department of Neurosurgery, Barrow Neurological Institute, St. Joseph's Hospital and Medical Center, Phoenix, Arizona
}

\begin{abstract}
Background: This study sought to compare index and adjacent-level biomechanics of cadaveric specimens with mature fusion versus normal spines in intact and acutely fused conditions.

Methods: Eight human cadaveric cervical spines with mature fusion across 1 to 3 levels were studied. Intervertebral angular range of motion (ROM) was determined at fused and adjacent levels during pure moments inducing flexion-extension (FE), lateral bending ( $\mathrm{LB}$ ), and axial rotation (AR). Mature fusion data were compared to data from normal spine specimens tested intact and then with a 1-level anterior plate/graft (fresh fixation). Bone qualities were compared using dual-energy x-ray absorptiometry.

Results: Mean bone mineral density was significantly greater in mature fusion spines $\left(0.632 \pm 0.239 \mathrm{~g} / \mathrm{cm}^{2}\right)$ than in normal spines $\left(0.489 \pm 0.195 \mathrm{~g} / \mathrm{cm}^{2}\right)(P<.001)$. Mean ROM for levels with mature fusion was $42 \%(\mathrm{FE}), 42 \%(\mathrm{LB})$, and $29 \%$ (AR) of the mean same-level ROM in freshly fixated specimens $(P \leq .045)$. The mean adjacent-level ROM in spines with mature fusion was less than in normal spines (matched levels) in all directions, with the greatest difference 1 level below fusion (FE: $-38 \%, P<.001$; LB: $-42 \%, P<.001$; AR: $-49 \%, P=.001$ ), followed by 1 level above fusion (FE: $-23 \%, P=.04$; LB: $-22 \%, P=.07$; AR: $-28 \%, P=.02)$ and 2 levels above fusion $(\mathrm{FE}:-20 \%, P=.08 ; \mathrm{LB}:-18 \%, P$ $=.11$; AR: $-31 \%, P=.009)$. Mature fusion reduced the magnitude of coupled LB during AR at C6-7 and C7-T1 $(P \leq$ $.03)$.
\end{abstract}

Conclusion: Cervical spine segments with mature fusion have higher bone mass, are less flexible than freshly fixed spines, and have reduced mobility at adjacent levels.

Cervical Spine

Keywords: adjacent segment, bone mass, cervical, mature fusion

\section{INTRODUCTION}

Cervical spine fusion procedures have been highly successful in treating spondylosis ${ }^{1-4}$; however, up to $25 \%$ of patients with good initial results experience further degenerative adjacent-segment disease (ASD) within 10 years. ${ }^{5}$ Whether these degenerative changes occur naturally or by altered load transfer across the adjacent discs is unknown. ${ }^{5-10}$

Although the etiology of ASD is most likely multifactorial, changes in load sharing and segmental motion have been implicated in its development. ${ }^{11}$ Some in vivo studies have suggested an immediate postoperative neck motion reduction after fusion; however, a progressive redistribution of motion throughout unfused levels may attenuate this effect, restoring or even increasing motion compared to the preoperative condition. ${ }^{12-16}$

Biomechanical studies of the effects of cervical fixation on index-level and adjacent-level motion can only simulate immediate postoperative mobility. ${ }^{17,18}$ Indeed, one standardized biomechanical testing protocol known as the hybrid testing protocol $^{19}$ has been entirely predicated on adjacent-segment hypermobility occurring immediately following surgery. However, this assumption has never been validated with substantial clinical evidence.

To our knowledge, a direct biomechanical comparison of motion at the index and adjacent levels between cadaveric spines with recent fresh fixation (nonfused) and those with mature fusion has not been studied in vitro and may provide further insight regarding immediate versus longterm range of motion (ROM) changes following fusion. The purpose of this in vitro study was to examine how cadaveric cervical spine specimens with mature fusion differ biomechanically from normal, freshly fixated cadaveric spine specimens at both the fused/instrumented and adjacent levels. 
Table 1. Mature fusion specimens.

\begin{tabular}{|c|c|c|c|c|c|c|c|}
\hline Specimen & Sex & Cause of Death & $\begin{array}{c}\text { Time Since } \\
\text { Fusion Surgery } \\
(\mathbf{y})\end{array}$ & $\begin{array}{c}\text { Age at Surgery } \\
(y)\end{array}$ & $\begin{array}{c}\text { Age at } \\
\text { Time of Death } \\
\text { (y) }\end{array}$ & Fused Levels & $\begin{array}{c}\text { No. of } \\
\text { Fused Levels }\end{array}$ \\
\hline 1 & $\mathrm{~F}$ & Brain tumor & 5 & 49 & 54 & C6-C7 & 1 \\
\hline 2 & $\mathrm{M}$ & Prostate cancer & 40 & 35 & 75 & C5-C6, no metal & 1 \\
\hline 3 & $\mathrm{~F}$ & Congestive heart failure & 20 & 67 & 87 & C4-C6 & 2 \\
\hline 4 & $\mathrm{~F}$ & Seizure, blood clot & 30 & 45 & 75 & C4-C5, no metal & 1 \\
\hline 5 & $\mathrm{~F}$ & Pneumonia & 10 & 67 & 77 & $\mathrm{C} 4-\mathrm{C} 7$ & 3 \\
\hline 6 & $\mathrm{~F}$ & Leiomyosarcoma & 15 & 34 & 49 & $\mathrm{C} 5-\mathrm{C} 7$ & 2 \\
\hline 7 & M & Lung cancer & 20 & 40 & 60 & $\mathrm{C} 5-\mathrm{C} 7$ & 2 \\
\hline 8 & $\mathrm{~F}$ & Cardiopulmonary arrest & 6 & 76 & 82 & $\mathrm{C} 4-\mathrm{C} 5$ & 1 \\
\hline Overall, mean (SD) & & & $18.3(12.1)$ & $51.6(16.2)$ & $69.9(13.8)$ & & $1.6(0.7)$ \\
\hline
\end{tabular}

Abbreviations: F, female; M, male.

\section{MATERIAL AND METHODS}

The flexibility of mature fusion specimens at index and adjacent levels was quantified as ROM, lax zone (LZ), and stiff zone (SZ). The ratio of main and coupled ROM during axial rotation (AR) and lateral bending (LB), referred to as coupling factor (CF), was also studied. The same parameters were studied in normal specimens tested intact and then with a 1-level anterior plate/graft (fresh fixation). Normal specimen data were pooled from specimens tested in the same laboratory and using the same methodology to allow level-by-level comparisons of index and adjacent levels in cervical spines with mature fusion and fresh fixation. Also, bone mineral densities (BMDs) of the mature fusion and normal spine segments were compared using dual-energy $\mathrm{x}$ ray absorptiometry (DEXA). Institutional review board approval was not required because of the nature of this cadaveric study.

\section{Specimens}

Eight cadaveric cervical spine specimens with mature fusion (Table 1) were collected fresh-frozen from a tissue bank with nationwide access to donor tissue. The collection time was almost 1 year because of the restrictive donor criteria of "cervical fusion within a known timeframe." The mean (SD) donor age was 69.9 (13.8) years at time of death and 51.6 (16.2) years at time of fusion. Mean (SD) time from fusion surgery to death was 18.3 (12.1) years. Intervertebral fusion with bone growth was confirmed on radiographs. For testing, specimens were stripped of muscle, keeping ligaments, joint capsules, and discs intact. Existing fusion hardware, if any, was left in place for mechanical testing and then carefully removed before DEXA.

Normal cervical specimens $(n=31)$, which were processed and tested in the same laboratory using identical testing methods, were grouped and served as controls for different parameters by providing comparative data for ROM, LZ, and SZ (intact and freshly fixated configurations); BMD; and/or CF. Specifically, mature fusion and normal specimens were grouped (Table 2) and assessed as follows:

- Group 1: mature fusion specimens obtained from the tissue bank and assessed for ROM, LZ, SZ, and CF (with hardware in place) and for BMD (after hardware removal), obtained from 6 women and 2 men.

- Group 2: control specimens from previous studies assessed exclusively for ROM, LZ, and SZ comparison while intact and after freshly fixated anterior fusion (C5-6), obtained from 5 women and 17 men.

- Group 3: control specimens from previous studies assessed exclusively for BMD comparison determined without hardware at levels matching those in group 1, obtained from 9 women and 15 men.

- Group 4: control specimens from previous studies assessed exclusively for CF comparison while intact and after freshly fixated anterior fusion (C5-6), obtained from 3 women and 4 men.

\section{Bone Quality Analysis}

Vertebral BMD at C3 through C7 (ie, 5 levels per specimen) was obtained anteroposteriorly using $1 \times$ $3.67-\mathrm{cm}$ regions of interest centered over the majority of vertebral bodies (groups 1 and 3). All scans were performed using a single clinical BMD scanner (Discovery W, Hologic, Waltham, Massachusetts). Mean BMD was determined per vertebral level in each group, and differences were compared using 1-way analysis of variance (ANOVA) fol- 
Table 2. Groups of specimens and spinal levels used for comparative analyses.

\begin{tabular}{|c|c|c|c|c|c|c|}
\hline \multirow[b]{2}{*}{ Group, Specimen(s) } & \multicolumn{6}{|c|}{ Cervical Spine Segments } \\
\hline & C2-3 & C3-4 & C4-5 & C5-6 & C6-7 & C7-T1 \\
\hline \multicolumn{7}{|l|}{ Group 1 , mature fusion $(\mathrm{n}=8)$} \\
\hline 1 & & & $(+2)$ & $(+1)$ & Fused & $(-1)$ \\
\hline 2 & & $(+2)$ & $(+1)$ & Fused & $(-1)$ & \\
\hline 3 & $(+2)$ & $(+1)$ & Fused & Fused & $(-1)$ & $(-2)$ \\
\hline 4 & $(+2)$ & $(+1)$ & Fused & $(-1)$ & $(-2)$ & \\
\hline 5 & $(+2)$ & $(+1)$ & Fused & Fused & Fused & $(-1)$ \\
\hline 6 & & $(+2)$ & $(+1)$ & Fused & Fused & $(-1)$ \\
\hline 7 & & $(+2)$ & $(+1)$ & Fused & Fused & $(-1)$ \\
\hline 8 & $(+2)$ & $(+1)$ & Fused & $(-1)$ & $(-2)$ & \\
\hline \multicolumn{7}{|l|}{ Group 2 , intact and then fresh anterior fixation $(\mathrm{n}=22)^{\mathrm{a}}$} \\
\hline C2-C7 specimens $(n=16)$ & & $(+2)$ & $(+1)$ & Fused & $(-1)$ & \\
\hline C2-T1 specimens $(n=6)$ & & $(+2)$ & $(+1)$ & Fused & $(-1)$ & $(-2)$ \\
\hline Group 3, DEXA $(\mathrm{n}=24)$ number of specimens per level & & 20 & 24 & 24 & 24 & \\
\hline Group 4, coupling factor $(n=7)$ number of specimens per level for comparison & & 7 & 7 & 7 & 7 & 7 \\
\hline
\end{tabular}

Abbreviations: $(+1)=1$ level above fusion; $(+2)=2$ levels above fusion; $(-1)=1$ level below fusion; $(-2)=2$ levels below fusion; DEXA $=$ dual-energy $\mathrm{x}$-ray absorptiometry.

${ }^{\mathrm{a}}$ Instrumentation for fresh anterior fixation includes cervical spine locking plate, Vectra-T, and Zero-P (Depuy Synthes, Raynham, Massachusetts)

lowed by Holm-Š́́dák pairwise tests, with $P$ values less than .05 considered significant.

\section{Biomechanical Testing}

Specimens were stored at $-20^{\circ} \mathrm{C}$ until test day and then thawed in normal saline at $21^{\circ} \mathrm{C}$. The distalmost vertebra was reinforced with household wood screws placed in a cylindrical metal mold and embedded using fast-curing resin (Smooth-Cast, Smooth-On, Easton, Pennsylvania) to permit attachment to the base of the testing apparatus (caudal end). The top vertebra was also reinforced with household screws and embedded in the same resin in a cylindrical pot $(\approx 200 \mathrm{~g})$ to attach to the test frame and loading fixture for load application (cranial end). Cables and pulleys imparted nondestructive, nonconstraining torque in conjunction with a standard uniaxial servohydraulic test frame (MTS Systems Corp., Minneapolis, Minnesota). ${ }^{20}$ Pure moment loading ensures that the same load is distributed to each level of the spine, allowing an equivalent comparison among all levels regardless of the distance from the point of loading. ${ }^{21}$ Loads were applied in $0.25-\mathrm{Nm}$ increments to a maximum of $1.5 \mathrm{Nm}$, with each load held 45 seconds to allow for creep.

Intervertebral motion was determined with the Optotrak 3020 camera system (Northern Digital, Inc., Waterloo, Ontario, Canada), which measured 3dimensional displacement of infrared-emitting markers rigidly attached to each vertebra. Custom software converted marker coordinates to angles about the local anatomical axes. ${ }^{22,23}$ Angle at maximum load (ROM) was separated into LZ, the portion of ROM in which the ligaments and hardware were lax, and SZ, the portion of ROM in which the ligaments and hardware were under tension. ${ }^{24}$

Mean CF was calculated as the ratio of coupled AR motion relative to primary $\mathrm{LB} \mathrm{ROM}\left(\mathrm{CF}_{\mathrm{AR} / \mathrm{LB}}\right)$ and coupled LB motion relative to primary AR $\mathrm{ROM}\left(\mathrm{CF}_{\mathrm{LB} / \mathrm{AR}}\right)$ at each level. The analysis of $\mathrm{CF}$ is important to better interpret the 3-dimensional motion of the cervical spine and its response to surgical interventions. Statistical comparisons among groups for ROM, LZ, SZ, and CF at index and adjacent levels were performed using 1-way ANOVA followed by Holm-Š́íák multiple comparison tests.

\section{RESULTS}

Mean (SD) donor age at death was significantly greater in group 1 (mature fusion, 69.9 [13.8] years) than in groups 2 (52.4 [11.5] years), 3 (53.6 [9.3] years), and 4 (48.9 [16.2] years) (normal) $(P<.001)$. The difference between mean (SD) age at time of fusion surgery in group 1 (51.6 [16.2] years) and donor age in groups 2, 3, and 4 was not statistically significant $(P=.27)$.

\section{Bone Quality (Group 1 vs Group 3)}

The mean (SD) BMD was significantly greater in spines with mature fusion (group 1, 0.632 [0.239] g/ $\mathrm{cm}^{2}$ ) than in normal spines (group 3, $0.489[0.195] \mathrm{g} /$ $\left.\mathrm{cm}^{2}\right)(P<.001)$. The differences in mean BMD (mature fusion versus intact) were more pronounced at levels above C7 (Figure 1), with a statistically significant difference at $\mathrm{C} 3(P=.02)$ and no difference at other levels. The BMD of fused versus 


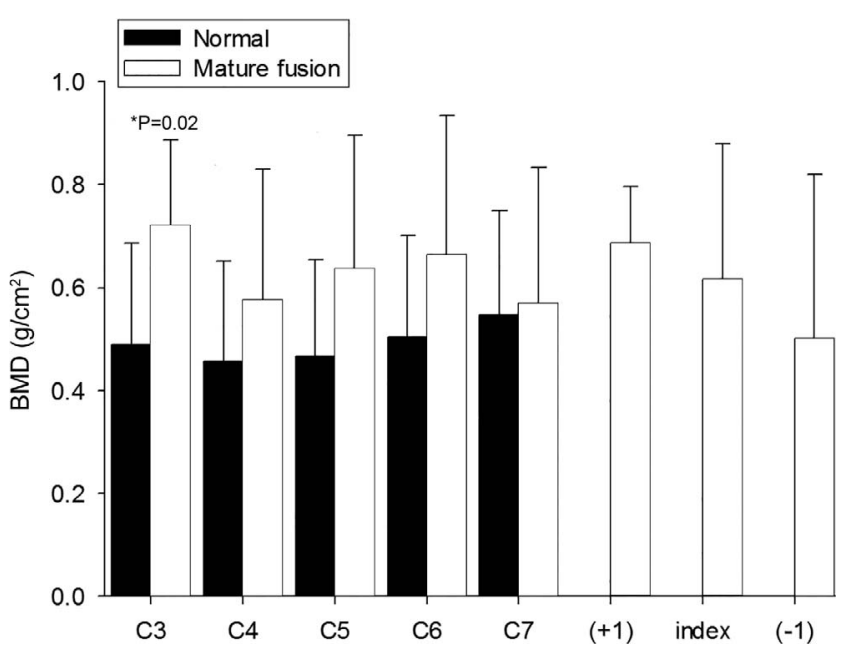

Figure 1. Mean bone mineral density (BMD) determined by means of dualenergy $\mathrm{x}$-ray absorptiometry analysis of different cervical levels of normal and mature-fusion specimens at $\mathrm{C} 3$ through $\mathrm{C} 7$. Error bars indicate standard deviation. Index denotes the fused levels, $(+1)$ denotes the adjacent level above the fused level, and $(-1)$ denotes the adjacent level below the fused level. The BMD at the $\mathrm{C} 3$ level was significantly greater in group $1(+1$ or +2 levels above mature fusion) than in group 3 (normal spines without fusion). Used with permission from Barrow Neurological Institute, Phoenix, Arizona.

adjacent vertebral levels did not differ in spines with mature fusion (group 1, $P=.61$; Figure 1).

ROM, LZ, and SZ (Group 1 Versus Group 2)

Index (Fused) Levels

To justify comparisons between mature fusion at multiple levels (C4-5, C5-6, and/or C6-7; group 1) and fresh fixation at only C5-6 (group 2), mean ROM, LZ, and SZ for the intact C5-6 level (group 2) were compared with those of intact C4-5, C5-6, and C6-7 levels combined (group 2); no differences were noted in any direction of loading (Figure 2, $P$ $\geq .52$ ). Mean ROM at levels with mature fusion (C4-5, C5-6, and/or C6-7, group 1) was significantly less than the mean ROM at the level with fresh fixation (group 2) in all directions of loading $(P \leq$ .045). Mean ROM at levels with mature fusion (group 1) was $42 \%$ of that for the instrumented level with fresh fixation (group 2) during FE (Figure 2, $P$ $=.02$ ), $42 \%$ during LB (Figure 2, $P=.045$ ), and $29 \%$ during AR (Figure 2, $P=.01$ ). Mean LZ at levels with mature fusion (group 1) was not different than that of the freshly fixated level in group 2 in any direction (Figure $2, P \geq .58$ ), whereas mean SZ was significantly smaller with mature fusion (Figure 2, $P<.001)$ than with fresh fixation for all directions.

\section{Adjacent Levels}

Mean ROM of group 2 (normal) specimens were not different before and after fusion at spinal levels adjacent $(+2,+1,-1$, or -2$)$ to the fresh fixation level in any direction (Figure $3, P \geq .61$ ). During $\mathrm{FE}$ and AR, mean ROM at both 1 level above and 1 level below a segment with mature fusion was significantly less than at the same levels in group 2

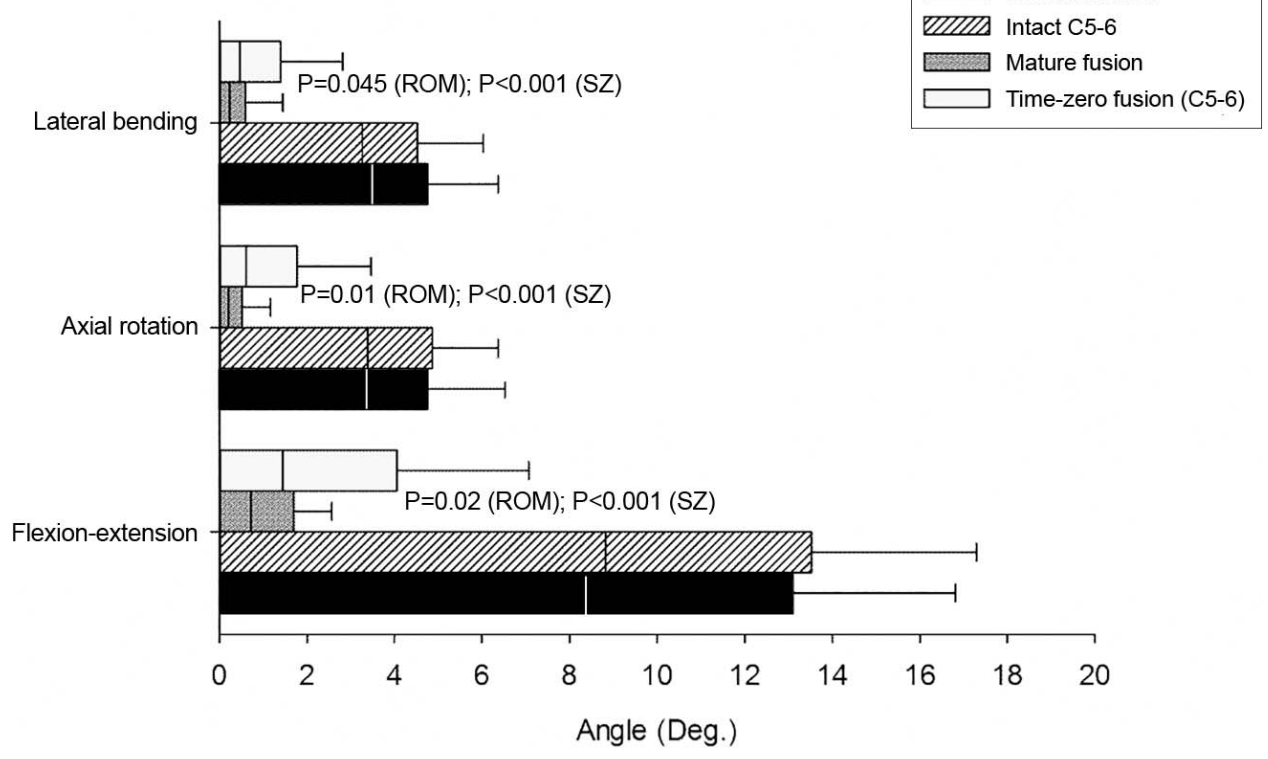

Figure 2. Graph showing mean range of motion (ROM; full bars), lax zone (from zero to the vertical dividing line), and stiff zone (SZ; from the vertical dividing line to ROM) at the index levels before and after fusion during each loading mode (lateral bending, axial rotation, flexion-extension). The $P$ values show level of significance from 1-way analysis of variance comparing intact levels (C5-6 alone in group 2 and C4-5, C5-6, and C6-7 combined in group 2), levels with mature fusion (C4-5, C5-6, and C6-7 combined in group 1), and levels with fresh fixation (C5-6 in group 2). Error bars indicate standard deviation of the ROM. Deg indicates degrees. Used with permission from [institution blinded for review]. 

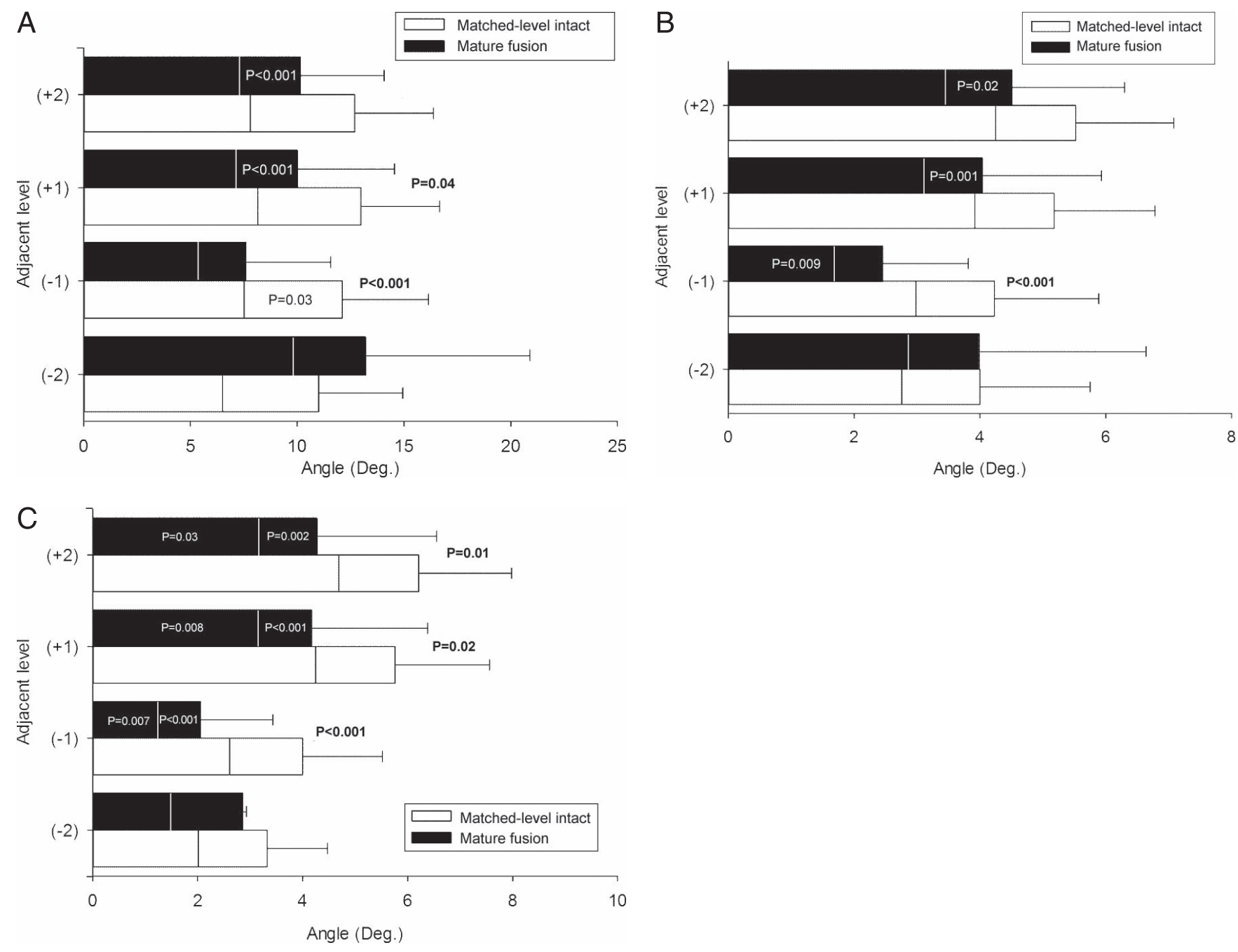

Figure 3. Graphs showing mean range of motion (ROM; full bars), lax zone ( $\mathrm{LZ}$; from zero to the vertical dividing line), and stiff zone (SZ; from the vertical dividing line to ROM) at the adjacent levels during (A) flexion-extension, (B) lateral bending, and (C) axial rotation. Two levels above fusion is indicated with (+2), 1 level above fusion is indicated with $(+1), 1$ level below fusion is indicated with $(-1)$, and 2 levels below fusion is indicated with ( -2$)$ (groups 1 and 2$)$. The $P$ values show level of significance (Student $t$ test) comparing levels adjacent to a fusion (group 1) and the same matched cervical levels from normal intact specimens (group 2). The $P$ values for $L Z$ and SZ are shown inside respective bars, and those for ROM are outside the bars. Error bars indicate standard deviation of the mean ROM. Deg indicates degrees. Used with permission from [institution blinded for review].

(Figures $3 \mathrm{~A}$ and $3 \mathrm{C}, P \leq .04)$. During LB, mean ROM at 1 level below a segment with mature fusion was significantly less than at the same levels in group 2 (Figure 3B, $P<.001$ ). At 1 level above fusion during LB, the difference between groups was not statistically significant $(P=.07)$. Mean ROM at 2 levels above a segment with mature fusion tended to be less than at the same levels in group 2. However, these differences were only statistically significant during AR (Figure 3C, $P=$ $.01)$. The difference at 2 levels below a segment with mature fusion compared with the same levels in group 2 was not significant for any direction (Figure $3, P \geq .77)$.

At both 1 and 2 levels above a mature fusion, ROM decreases were attributable to SZ decreases more than to $\mathrm{LZ}$ decreases, with $\mathrm{SZ}$ significantly less in group 2 at both 1 and 2 levels above fusion for all directions of motion (Figure $3, P \leq .02$ ). The LZs at 1 and 2 levels above mature fusion (group 1) were not different from the same levels in group 2 during FE and LB (Figures $3 \mathrm{~A}$ and $3 \mathrm{~B}, P$ $\geq$.16). During AR, mean $\mathrm{LZ}$ was less at 1 level above $(P=.008)$ and 2 levels above $(P=.03)$, compared with similar levels in group 2 (Figure 3C). At 1 level below mature fusion, mean LZ was less than at the same levels in group 2 during LB and AR (Figures 3B and 3C, $P \leq .009$ ) but not statistically significantly different during FE (Figure $3 \mathrm{~A}, P=.06$ ). Both $\mathrm{SZ}$ and $\mathrm{LZ}$ remained unchanged at 2 levels below mature fusion (Figure $3, P \geq .11)$. 

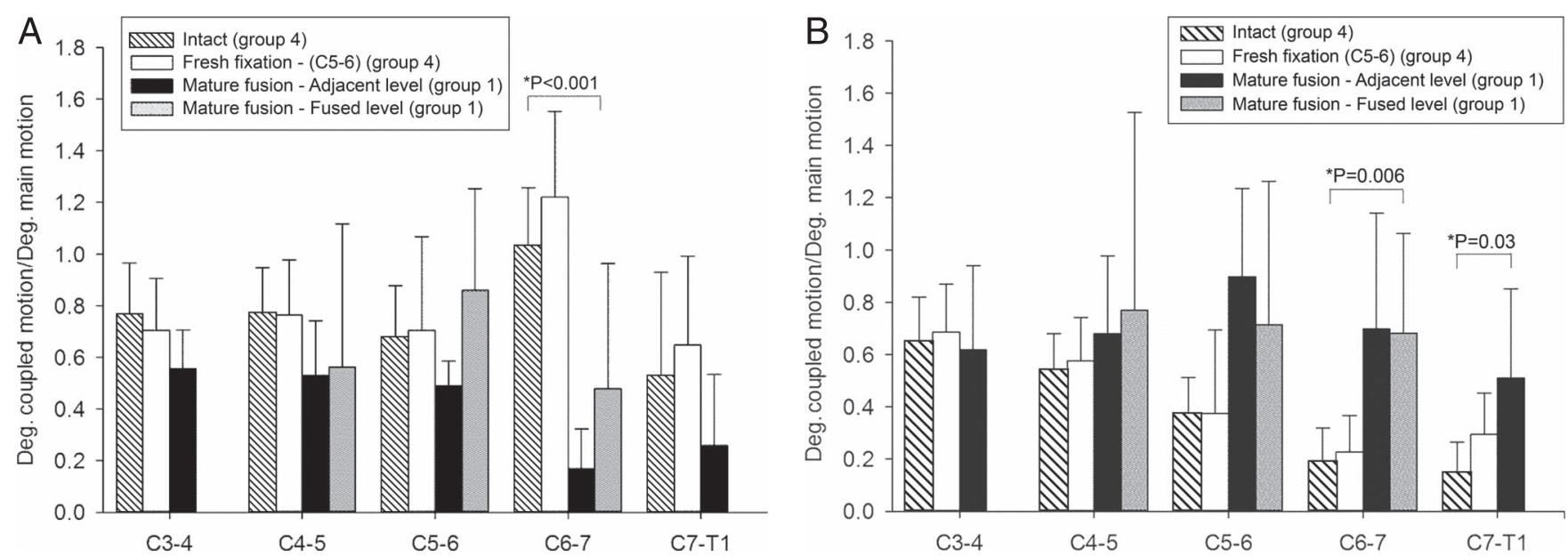

Figure 4. Angular coupled rotation per degree of primary rotation showing coupling patterns between lateral bending and axial rotation. (A) Lateral bending during axial rotation. (B) Axial rotation during lateral bending at different levels. Two levels above fusion is indicated with $(+2), 1$ level above fusion is indicated with $(+1), 1$ level below fusion is indicated with $(-1)$, and 2 levels below fusion is indicated with $(-2)$. Error bars indicate standard deviation. Deg indicates degrees. Used with permission from [institution blinded for review].

\section{Coupling Factors (Group 1 Versus Group 4)}

Differences between groups 1 and 4 were statistically significant in terms of coupling patterns at C6-7 (Figure 4, $P<.001$ for mean $\mathrm{CF}_{\mathrm{LB} / \mathrm{AR}}, P=$ .006 for mean $\left.\mathrm{CF}_{\mathrm{AR} / \mathrm{LB}}\right)$ and $\mathrm{C} 7-\mathrm{T} 1(P=.03$ for mean $\left.\mathrm{CF}_{\mathrm{AR} / \mathrm{LB}}\right)$. Groups 1 and 4 did not differ significantly in mean coupling pattern $\left(\mathrm{CF}_{\mathrm{LB} / \mathrm{AR}}\right.$ or $\left.\mathrm{CF}_{\mathrm{AR} / \mathrm{LB}}\right)$ at $\mathrm{C} 3-4, \mathrm{C} 4-5$, or C5-6 $(P \leq .14)$.

\section{DISCUSSION}

\section{Bone Quality}

Patients who require spine surgery often have low BMD as a consequence of older age. ${ }^{25}$ Previously our laboratory retrospectively studied the relationship between parameters including age, BMD, ROM, LZ, and SZ in 285 intact cadaveric lumbar motion segments ${ }^{26}$ and in 581 intact cervical cadaveric motion segments. ${ }^{27}$ Both studies observed a significant negative correlation between BMD and increased age $(P \leq .02)$. Nevertheless, in the current study, mean BMD was greater in spines with mature fusion than in intact normal spines, despite the greater mean age of mature fusion specimens (69.9 [13.8] versus 53.6 [9.3] years). This finding supports the hypothesis that the fusion bone mass ensures increased density when compared to nonfused spines.

Others have shown that the fusion bone mass usually has better bone quality than its tissue of origin (eg, iliac crest), indicating that weaker bone responds to the new loading environment with cortical and trabecular hypertrophy as well as increased mineralized volume, which suggests adaptive bone remodeling. ${ }^{28} \mathrm{We}$ observed higher BMD values in mature fused cervical spines at both the fused and adjacent levels.

\section{ROM, LZ, and SZ}

\section{Index (Fused) Level}

One of our main findings is the decrease in mobility $(58 \%-71 \%)$ at levels with mature fusion compared with fresh fixation levels (group 2). To our knowledge, this is the first in vitro study involving a rare set of human spine segments that quantifies the decrease in mobility of spinal levels with mature fusion versus those fixated with spinal instrumentation, which provides insight regarding the differences between the two. Thus, although initial instrumentation fixation can provide significant reductions in ROM in spine segments to promote fusion, a fully fused bony bridge clearly further significantly improves spinal stability.

\section{Adjacent Level}

ROM at levels adjacent to fresh fixation was not significantly different before and after fusion in any direction of loading. This is expected with pure moment loading: fusing 1 level should not affect adjacent-level stiffness because the bending moment at each level remains constant. A primary finding in this study, however, was that ROM at both 1 level above $(+1)$ and 1 level below (-1) a segment with mature fusion was significantly less than that of the same levels in specimens without mature fusion. In addition, ROM at 2 levels above $(+2)$ a segment 
with mature fusion was significantly less than that of the same levels in specimens without mature fusion (Figure $3 \mathrm{C}, P=.01$ for AR).

In other words, we observed significant reductions in mobility at both levels $(+1$ and -1$)$ that were adjacent to mature fusion, with greater differences at caudal versus rostral adjacent levels. We also observed reductions at 2 levels rostral to mature fusion. Changes in ROM at levels adjacent to mature fusion were noted as attributable to alterations in SZ rather than LZ, suggesting that soft tissues (discs and/or ligaments) at adjacent levels become stiffer over time.

Both of the above-mentioned retrospective studies conducted in our laboratory generally demonstrated no significant correlation between ROM and specimen age, ${ }^{26,27}$ but significant negative correlations in ROM with increased BMD in both lumbar $(P \leq .04)$ and cervical $(P \leq .01)$ spines in $\mathrm{FE}$ and LB. The SZ was significantly negatively correlated with increased age in both spine regions in FE $(P<$ $.001)$ and LB $(P<.001)$ but not with BMD. In contrast, the LZ did not correlate with age but significantly negatively correlated with increased BMD in the cervical spine in FE $(P=.01)$ and $\mathrm{LB}(P$ $=.01$ ). In the current study, the SZ was noted to be significantly reduced in at least 2 adjacent levels in all directions of testing. With the exception of the second level caudal to a mature fusion, mean LZ values in the current study were smaller in mature fusion specimens with significant differences noted in LB and AR. Collectively, these observations indicate that the reduced ROM observed at adjacent levels cannot be explained by the increased age of this group alone, but is also related to the increased BMD levels of these specimens that are inconsistent with increased age alone.

A common expectation is that biomechanical testing can predict adjacent-segment hypermobility after fusion. This expectation may result from data collected under the hybrid test protocol. ${ }^{19}$ In this protocol, intact specimens are first subjected to a nondestructive applied pure moment load, and the resulting global ROM is recorded. Subsequently, specimens with fixated spinal levels are forced to the same global intact ROM, forcing nonfixated levels to increase their contribution to achieve the same global displacement as the intact spine segment.

Past clinical reviews of preoperative and postoperative motion of the lumbar spine, however, have indicated that excessive motion at adjacent segments after spinal fusion generally does not occur, and that global spinal ROM tends to decrease after fusion. ${ }^{29}$ In this study, under pure moment loading, maturely fused cervical specimens importantly also demonstrated no evidence of adjacent-segment hypermobility, providing in vitro biomechanical evidence that global whole-neck motion would most likely be reduced following fusion surgery. Significant concerns have been documented with the hybrid protocol based on its underlying assumption of maintained global ROM and on the excessive loads often needed to drive a fixated spine segment to the same global ROM as the intact spine segment. ${ }^{29-31}$ Further studies are necessary to better characterize postfusion global neck motion and its time course. It is possible that adaptations in neck mobility are an individual phenomenon without the possibility to be collectively determined given the presence of several variables (age, sex, presence of osteoarthritis in other levels, or adjacent-level ligament compliance).

Whether ASD after fusion is a postoperative complication due to mechanical changes or a progression of the natural history of cervical degeneration remains unclear and a highly controversial topic. ${ }^{32,33}$ Changes in the mechanical demand can lead to changes in intradiscal pressures and, consequently, changes in nutrient diffusion forces. Given that the disc is avascular, altered intradiscal pressure can lead to unbalanced nutrition, accumulation of metabolic waste, decreased $\mathrm{pH}$, and finally cell death. It is well established that cell death is involved in ASD pathogenesis; however, the trigger remains unknown. The cause of cell death is most likely multifactorial, with altered biomechanical stresses at the adjacent level in addition to surgical disruption of associated ligaments and joint capsules, postoperative misalignments, age, smoking, and the natural history of the disease. $^{34}$

\section{Coupling Factors}

The gradual decrease in $\mathrm{CF}_{\mathrm{AR} / \mathrm{LB}}$ along the cervical spine found in group 2 specimens (intact with fresh fixation) is consistent with the literature: 0.67 at $\mathrm{C} 2-3$ to 0.13 at $\mathrm{C} 7-\mathrm{T} 1 .^{21}$ Crawford et $\mathrm{al}^{24}$ reported $\mathrm{CF}_{\mathrm{AR} / \mathrm{LB}}$ values of 0.70 (intact) and 0.28 (fresh fixation) at C4-5, compared with 0.54 (intact) at $\mathrm{C} 4-5$ in our study. In contrast with Crawford et $\mathrm{al},{ }^{24}$ who noted a decrease in $\mathrm{CF}_{\mathrm{AR} / \mathrm{LB}}$ after anterior 
fusion at $\mathrm{C} 4-5$, we did not note a change in $\mathrm{CF}_{\mathrm{AR} / \mathrm{LB}}$ after fresh fixation at C5-6. Nonetheless, in terms of $\mathrm{CF}_{\mathrm{AR} / \mathrm{LB}}$, we found a significant difference between fresh fixation and mature fusion at C6-7 and C7-T1. Changes in kinematic parameters such as the $\mathrm{CF}$ may indicate that the load distribution at levels adjacent to fusion was affected by fusion.

\section{Limitations}

Cadaveric studies have inherent limitations, including the absence of contributions of the paraspinal muscles, which play a key role in spinal movement in vivo, as well as assessment of immediate acute stabilization only with spinal fixation. Because the control-specimen data originated from previous studies using similar methods, the entire sample was divided into 4 groups, 1 for mature fusion and 1 more for controls for each parameter (ROM, BMD, and $\mathrm{CF}$ ). Not all previous studies had collected all parameters at once.

Selecting donors with previous cervical spine fusions unrelated to the cause of death among tissue banks is itself a very challenging task. Achieving uniformity on donors' procedures in terms of number of levels treated, approaches chosen, type of implant, graft utilized, and reason for surgical indications was not practically feasible. The heterogenicity of the mature fusion samples could not be avoided and should be considered when interpreting the results of the study; however, the novelty of the purpose of the study warranted proceeding with this limitation. Furthermore, the mature fusion stiffness depended more on the individual bone mass quality formed than the type or time of fixation itself. Also, the presence of systemic diseases in some donors could affect bone quality and mobility; however, this effect was diminished by routinely screening all donors to exclude evident local pathologies. Lastly, in vitro cadaveric biomechanical findings have not always consistently correlated with the in vivo clinical scenario. Although this discrepancy may be due in part to the inherent limitations of cadaver studies, adoption of the hybrid testing protocol (ie, an in vitro test method that intrinsically produces adjacentsegment hypermobility in the setting of spinal fusion) has likely contributed to this disconnect. Further studies are necessary to correlate in vitro adjacent-level biomechanical changes with clinical symptoms and postoperative outcomes.

\section{CONCLUSION}

Human cadaveric cervical spinal segments with mature fusion have greater bone mass and less mobility than cervical spines with fresh fixation. Mobility of a cervical spinal level with mature fusion is less than half that of a level with anterior plate fixation immediately after surgery. Under pure moment loading, maturely fused cervical specimens demonstrated no evidence of adjacent-segment hypermobility. Mobility at immediately adjacent $( \pm 1)$ levels decreases significantly over the long term, with greater differences caudally.

\section{ACKNOWLEDGMENTS}

In memoriam: Bruce Dean, MD. The authors gratefully acknowledge Luis Perez-Orribo, MD, $\mathrm{PhD}$, for his contribution to the collection and analysis of the data, and the staff of Neuroscience Publications at Barrow Neurological Institute for assistance with manuscript preparation.

\section{REFERENCES}

1. Hacker RJ. A randomized prospective study of an anterior cervical interbody fusion device with a minimum of 2 years of follow-up results. J Neurosurg. 2000;93(2 suppl):222226.

2. Samartzis D, Shen FH, Lyon C, Phillips M, Goldberg EJ, An HS. Does rigid instrumentation increase the fusion rate in one-level anterior cervical discectomy and fusion? Spine $J$. 2004;4(6):636-643.

3. Kaiser MG, Haid RW Jr, Subach BR, Barnes B, Rodts GE Jr. Anterior cervical plating enhances arthrodesis after discectomy and fusion with cortical allograft. Neurosurgery. 2002;50(2):229-236; discussion 236-228.

4. Samartzis D, Shen FH, Goldberg EJ, An HS. Is autograft the gold standard in achieving radiographic fusion in one-level anterior cervical discectomy and fusion with rigid anterior plate fixation? Spine (Phila Pa 1976). 2005;30(15):1756-1761.

5. Hilibrand AS, Carlson GD, Palumbo MA, Jones PK, Bohlman HH. Radiculopathy and myelopathy at segments adjacent to the site of a previous anterior cervical arthrodesis. $J$ Bone Joint Surg Am. 1999;81(4):519-528.

6. Emery SE, Bolesta MJ, Banks MA, Jones PK. Robinson anterior cervical fusion comparison of the standard and modified techniques. Spine (Phila Pa 1976). 1994;19(6):660 663.

7. Goffin J, van Loon J, Van Calenbergh F, Plets C. Longterm results after anterior cervical fusion and osteosynthetic stabilization for fractures and/or dislocations of the cervical spine. J Spinal Disord. 1995;8(6):500-508; discussion 499.

8. Gore DR, Sepic SB. Anterior cervical fusion for degenerated or protruded discs. A review of one hundred forty-six patients. Spine (Phila Pa 1976). 1984;9(7):667-671.

9. Herkowitz HN, Kurz LT, Overholt DP. Surgical 
management of cervical soft disc herniation. A comparison between the anterior and posterior approach. Spine (Phila Pa 1976). 1990;15(10):1026-1030.

10. Hilibrand AS, Robbins M. Adjacent segment degeneration and adjacent segment disease: the consequences of spinal fusion? Spine J. 2004;4(6 suppl):190S-194S.

11. Saavedra-Pozo FM, Deusdara RA, Benzel EC. Adjacent segment disease perspective and review of the literature. Ochsner J. 2014;14(1):78-83.

12. Reitman CA, Hipp JA, Nguyen L, Esses SI. Changes in segmental intervertebral motion adjacent to cervical arthrodesis: a prospective study. Spine (Phila Pa 1976). 2004;29(11):E221-226.

13. Chien A, Lai DM, Wang SF, Hsu WL, Cheng CH, Wang JL. Comparison of cervical kinematics, pain, and functional disability between single- and two-level anterior cervical discectomy and fusion. Spine (Phila Pa 1976). 2016;41(15):E915-922.

14. Chien A, Lai DM, Wang SF, Cheng CH, Hsu WL, Wang JL. Differential segmental motion contribution of single- and two-level anterior cervical discectomy and fusion. Eur Spine J. 2015;24(12):2857-2865.

15. Hilibrand AS, Balasubramanian K, Eichenbaum M, et al. The effect of anterior cervical fusion on neck motion. Spine (Phila Pa 1976). 2006;31(15):1688-1692.

16. Wu XD, Wang XW, Yuan W, et al. The effect of multilevel anterior cervical fusion on neck motion. Eur Spine J. 2012;21(7):1368-1373.

17. Eck JC, Humphreys SC, Lim TH, et al. Biomechanical study on the effect of cervical spine fusion on adjacent-level intradiscal pressure and segmental motion. Spine (Phila $\mathrm{Pa}$ 1976). 2002;27(22):2431-2434.

18. Kretzer RM, Hsu W, Hu N, et al. Adjacent-level range of motion and intradiscal pressure after posterior cervical decompression and fixation: an in vitro human cadaveric model. Spine (Phila Pa 1976). 2012;37(13):E778-E785.

19. Panjabi MM. Hybrid multidirectional test method to evaluate spinal adjacent-level effects. Clin Biomech (Bristol, Avon). 2007;22(3):257-265.

20. Crawford NR, Brantley AG, Dickman CA, Koeneman EJ. An apparatus for applying pure nonconstraining moments to spine segments in vitro. Spine (Phila Pa 1976). 1995;20(19):2097-2100.

21. White AA, III, Panjabi MM. Clinical Biomechanics of the Spine. 2nd ed. Philadelphia, PA: Lippincott; 1990.

22. Crawford NR, Dickman CA. Construction of local vertebral coordinate systems using a digitizing probe. Technical note. Spine (Phila Pa 1976). 1997;22(5):559-563.

23. Crawford NR, Yamaguchi GT, Dickman CA. Methods for determining spinal flexion/extension, lateral bending, and axial rotation from marker coordinate data: analysis and refinement. Hum Movement Sci. 1996;15(1):55-78.

24. Crawford NR, Baek S, Sawa AG, Safavi-Abbasi S, Sonntag VK, Duggal N. Biomechanics of a fixed-center of rotation cervical intervertebral disc prosthesis. Int J Spine Surg. 2012;6:34-42.

25. Chin DK, Park JY, Yoon YS, et al. Prevalence of osteoporosis in patients requiring spine surgery: incidence and significance of osteoporosis in spine disease. Osteoporos Int. 2007;18(9):1219-1224.

26. Sawa AGU, Lehrman JN, Crawford NR, Kelly BP. Variations among human lumbar spine segments and their relationships to in vitro biomechanics: a retrospective analysis of 281 motion segments from 85 cadaveric spines. Int $J$ Spine Surg. 2020;14(2):140-150.

27. Newcomb AG, Lehrman J, Andrada B. Variations among human spine segments and their relationships to in vitro kinematics: a retrospective analysis of experimental data including 581 cervical motion segments from 147 donor spines. Paper presented at; ISASS18; September 26, 2018; Toronto, Canada.

28. Kleiner JB, Odom JA Jr, Moore MR, Wilson NA, Huffer WE. The effect of instrumentation on human spinal fusion mass. Spine (Phila Pa 1976). 1995;20(1):90-97.

29. Malakoutian M, Volkheimer D, Street J, Dvorak MF, Wilke HJ, Oxland TR. Do in vivo kinematic studies provide insight into adjacent segment degeneration? A qualitative systematic literature review. Eur Spine J. 2015;24(9):1865-1881.

30. Kelly BP, Theodore N. What can standard in vitro biomechanical testing tell us about adjacent segment disease? World Neurosurg. 2016;89:710-712.

31. Crawford NR. Does the "hybrid multidirectional test method" generate quality data or paradoxical data? Clin Biomech (Bristol, Avon). 2007;22(7):861-864.

32. Alhashash M, Shousha M, Boehm H. Adjacent segment disease after cervical spine fusion: evaluation of a 70-patient long-term follow-up. Spine (Phila Pa 1976). 2018;43(9):605609.

33. Jack A, Hardy St-Pierre G, Nataraj A. Adjacent segment pathology: progressive disease course or a product of iatrogenic fusion? Can J Neurol Sci. 2017;44(1):78-82.

34. Chung JY, Park JB, Seo HY, Kim SK. Adjacent segment pathology after anterior cervical fusion. Asian Spine $J$. 2016;10(3):582-592.

Disclosures and COI: The authors report no conflicts of interest regarding presented methods or findings. No external research grants were received to support this work, which was internally funded.

Corresponding Author: Brian P. Kelly, PhD, c/o Neuroscience Publications; Barrow Neurological Institute, St. Joseph's Hospital and Medical Center, 350 W. Thomas Road; Phoenix, AZ 85013. Phone: (602) 406-3593; Fax: (602) 406-4104; Email: Neuropub@barrowneuro.org.

Published 0 Month 2021

This manuscript is generously published free of charge by ISASS, the International Society for the Advancement of Spine Surgery. Copyright (C) 2021 ISASS. To see more or order reprints or permissions, see http://ijssurgery.com. 